\title{
Coupled Experiment/Simulation Approach for the Design of Radiation-Hardened Rare-Earth Doped Optical Fibers and Amplifiers
}

\author{
S. Girard, Member, IEEE, L. Mescia, M. Vivona, A. Laurent, Y. Ouerdane, C. Marcandella, \\ F. Prudenzano, A. Boukenter, T. Robin, P. Paillet, Senior Member, IEEE, \\ V. Goiffon, Member, IEEE, B. Cadier, M. Cannas, and R. Boscaino
}

\begin{abstract}
We developed an approach to design radiationhardened rare earth -doped fibers and amplifiers. This methodology combines testing experiments on these devices with particle swarm optimization (PSO) calculations. The composition of $\mathrm{Er} / \mathrm{Yb}$-doped phosphosilicate fibers was improved by introducing Cerium inside their cores. Such composition strongly reduces the amplifier radiation sensitivity, limiting its degradation: we observed a gain decreasing from $19 \mathrm{~dB}$ to $18 \mathrm{~dB}$ after $50 \mathrm{krad}$ whereas previous studies reported higher degradations up to $0^{\circ} \mathrm{dB}$ at such doses. PSO calculations, taking only into account the radiation effects on the absorption efficiency around the pump and emission wavelengths, correctly reproduce the general trends of experimental results. This calculation tool has been used to study the influence of the amplifier design on its radiation response. The fiber length used to ensure the optimal amplification before irradiation may be rather defined and adjusted to optimize the amplifier performance over the whole space mission profile rather than before integration in the harsh environments. Both forward and backward pumping schemes lead to the same kind of degradation with our active fibers. By using this promising coupled approach, radiation-hardened amplifiers nearly insensitive to radiations may be designed in the future.
\end{abstract}

Index Terms - radiation effects, optical fibers, rare-earth ions, amplifiers, particle swarm optimization, erbium, ytterbium.

\section{INTRODUCTION}

$\mathrm{R}$ ADIATION responses of Rare-Earth (RE) doped optical fibers are widely studied since these waveguides are a key element of fiber-based systems such as amplifiers or lasers with high power capabilities. Such active fibers were also shown to be the most sensitive part of these systems to

Manuscript received September $16^{\text {th }}, 2011$.

S. Girard and C. Marcandella, P. Paillet are with CEA, DAM, DIF, F91297 Arpajon France ().

L. Mescia is with Dipartimento di Elettrotecnica ed Elettronica, Politecnico di Bari, I-70125 Bari, Italy.

M. Vivona, A. Boukenter, Y. Ouerdane are with University of Saint-Etienne, Laboratoire Hubert Curien, UMR CNRS 5516, F42000 Saint-Etienne, France. M. Vivona, A. Laurent, T. Robin, B. Cadier are with IXFiber SAS, F-22300 Lannion, France.

F. Prudenzano is with Dipartimento di Ingegneria dell'Ambiente e per lo Sviluppo Sostenibile, I-74100 Taranto, Italy.

V. Goiffon is with Université de Toulouse, ISAE, F-31055 Toulouse, France.

M. Vivona, M. Cannas and R. Boscaino are with Dipartimento di Scienze Fisiche ed Astronomiche dell’Università di Palermo, I-90123, Italy. radiations [1-3]. As a consequence and despite the short length used for space applications (typically few meters), the estimation of their vulnerability to the harsh environment associated with space missions remains crucial [4-5]. Most of the previous studies, including those of our research group, have been devoted to the characterization of their radiation response in a passive configuration (without pumping of the active ions involved) with test benches comparable to the ones used to characterize of Telecom grade optical fibers [1-7].

Origins of the radiation-induced attenuation (RIA) measured during passive configuration tests have now been partially identified. For most of the RE-doped fibers, such as those containing aluminum $(\mathrm{Al})$ or phosphorus $(\mathrm{P})$ in their silicabased cores, the point defects at the origin of their degradation seem rather related to the host glass matrix than to RE ions [1, 8]. We also recently showed, by comparing Er- and Er/Ybdoped fibers, that the codoping with the $\mathrm{Yb}^{3+}$ ions did not increase the Er-doped fiber radiation sensitivity [8]. As a consequence, the $\mathrm{Yb} / \mathrm{Er}$ composition allows obtaining higher amplification gain compared to Er-doped fibers and similar radiation behaviors than Er-doped glasses. Furthermore, we showed, in [8], that the competition between the trapping of charges released by irradiation in the matrix may be used to design fibers less sensitive to radiations.

From these previous studies in passive configuration, Er/Ybcodoped optical fibers are identified as the most promising candidates for the design of radiation-hardened amplifiers. Fewer studies have been devoted to the characterization of $\mathrm{Er} / \mathrm{Yb}$-doped fibers in an active configuration, even less as part of fiber-based amplifiers. The responses of optically pumped RE-doped fibers and amplifiers have been discussed in [9-12]. In [12], an important degradation of an $\mathrm{Er} / \mathrm{Yb}$ amplifier output power with cumulated dose was shown. In this work, M. Alam et al. showed a complete darkening of their amplifier (extinction of the amplified signal gain) after a dose of $20 \mathrm{krad}$ at the dose rate of $20 \mathrm{rad} / \mathrm{s}$ or after a dose of $40 \mathrm{krad}$ at a lower dose rate of $10 \mathrm{rad} / \mathrm{s}$. Jin Ma et al. [13] measured the complete darkening of the output power of their $\mathrm{Er} / \mathrm{Yb}$ amplifier after a $50 \mathrm{krad}$ dose at a dose rate of $40 \mathrm{rad} / \mathrm{s}$.

In this paper, we present a new coupled experiment/simulation approach that we built to design radiation-hardened fiberbased amplifiers for space applications. First, we experimentally validate the definition of a radiation-hardened 
fiber thanks to our understanding about radiation effects on RE-doped glasses [1, 8]. This Er/Yb-doped optical fiber has been made by Ixfiber SAS, and is hereafter called fiber J. This new fiber has been used to design and investigate a radiationtolerant optical amplifier, named A\#2. The characterization of this amplifier and the comparison with the results obtained with a standard $\mathrm{Er} / \mathrm{Yb}$ fiber (hereafter called fiber I) are not fully presented as this in situ response is more detailed in [14]. In this paper, we focused on the second part of our coupled approach: the hardening by design of the amplifier. For this, a homemade computer code based on particle swarm optimization (PSO) approach and rate equations model has been built to simulate both the spectroscopic properties of active ions inside the fiber core and then predict the amplifier performance before, during and after irradiation. We demonstrate the ability of the developed computer code to correctly simulate the amplifier behavior in a harsh environment. After validation, we illustrate the general trends that can be applied to imagine the design of optical amplifiers virtually insensitive to radiations.

\section{EXPERIMENT/SIMULATION PROCEDURE}

\section{A. Tested Optical Fibers and Amplifiers}

Two prototype fibers doped with similar concentrations of $\mathrm{Er}^{3+}$ and/or $\mathrm{Yb}^{3+}$ ions in their phosphosilicates cores have been developed by Ixfiber SAS. These fibers, named I and J, were designed on the basis of our previous investigations that allowed us to put forward new designs for RE-doped fibers to improve their radiation tolerances. They have bare fiber geometry with an octagonal double-clad (DC) which is designed for easier coupling of the high power multimode pump radiation into the RE-doped cores (see inset of Fig.1.). This DC is made of pure-silica glass and is similar for the two waveguides. The main difference between the two fibers is the addition of Cerium (Ce) ions in the core of fiber J. As expected from our previous work [14-15], this element strongly affects the radiation sensitivity of RE-doped glasses without degrading its amplification performance.

Fig.1 illustrates the attenuation spectra of the two fibers before irradiation, measured by the cut-back method through excitation of the DC in the multimode regime with a white light source. In this figure, we observe the strong absorption bands related to the RE-ions around the pump wavelength $\left(915 \mathrm{~nm}, \mathrm{Yb}^{3+}\right.$ ions) and the emission wavelength $(1545 \mathrm{~nm}$, $\mathrm{Er}^{3+}$ ions).

Based on I \& J active fibers, two amplifiers with comparable performances were designed and fully characterized, called

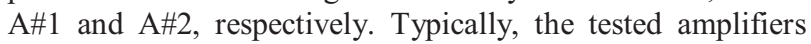
exhibited a $19 \mathrm{~dB}$ gain with a $10 \mathrm{dBm}$ input power. To obtain such performance; we have used $12 \mathrm{~m}$ of RE-doped fiber and a backward pumping scheme. In this configuration, the $915 \mathrm{~nm}$ pump laser and the $1545 \mathrm{~nm}$ signal to be amplified propagate in opposite directions inside the fiber, improving the amplifier performance [16]. The output power at $1545 \mathrm{~nm}$ was limited to less than $1 \mathrm{~W}$ for these experiments but this

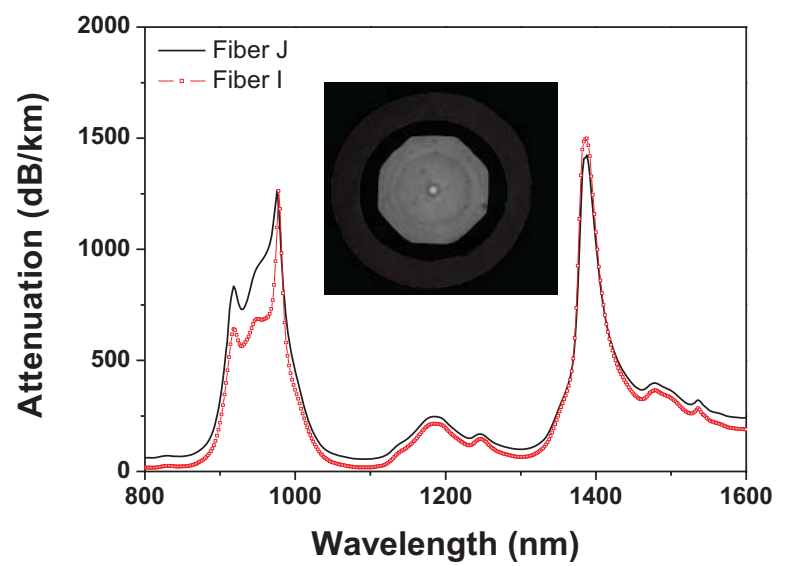

Fig. 1. Background attenuation measured by the cut-back method in fibers I and $\mathrm{J}$ through excitation of the DC with a white light source. The inset illustrates a picture of $\mathrm{J}$ fiber.

amplifier design can easily extract up to $10 \mathrm{~W}$ with sufficient pump and input signal power available.

\section{B. Radiation Tests}

Two different irradiation campaigns have been performed in the framework of this study. The first one took place in 2010 at the Co60 source from CEA in Bruyères-le-Châtel and was devoted to the in situ characterization of the radiation responses of the two amplifiers A\#1 and A\#2 under $\gamma$-rays $(1.2 \mathrm{MeV})$. These experiments were conducted at a low dose rate of $\sim 0.3 \mathrm{rad} / \mathrm{s}$, considered cumulative doses limited to $\sim 50 \mathrm{krad}$ and room temperature. These first experiments proved the strong interest of codoping the Er/Yb-doped fiber core with $\mathrm{Ce}$ [14]. An important point was also highlighted during this first campaign as the radiation-induced degradation of our two amplifiers, in terms of radiation-induced attenuation (RIA), remains stable after irradiation at room temperature.

The second campaign was performed in 2011 at UCL, University in Louvain-la-Neuve, Belgium. During these tests, different samples of the two I and J fibers have been irradiated at several doses from 7 to $70 \mathrm{krad}$ at a dose rate of about $\sim 0.3 \mathrm{rad} / \mathrm{s}$ without monitoring of the fiber response during irradiation. This experiment was devoted to post mortem measurements of the radiation-induced changes directly in the two I and J fibers, e-g. attenuation values around the pump and emission wavelengths. These extracted values, which could not be obtained from amplifier testing, are mandatory input parameters for the PSO calculations implied in our simulation approach.

\section{Fiber and Amplifier Simulations}

Up to now, no simulation study was performed to improve the radiation resistance of fiber-based amplifiers. However, some existing models reproduce the mechanisms leading to the infrared signal amplification in RE-doped optical fibers. 


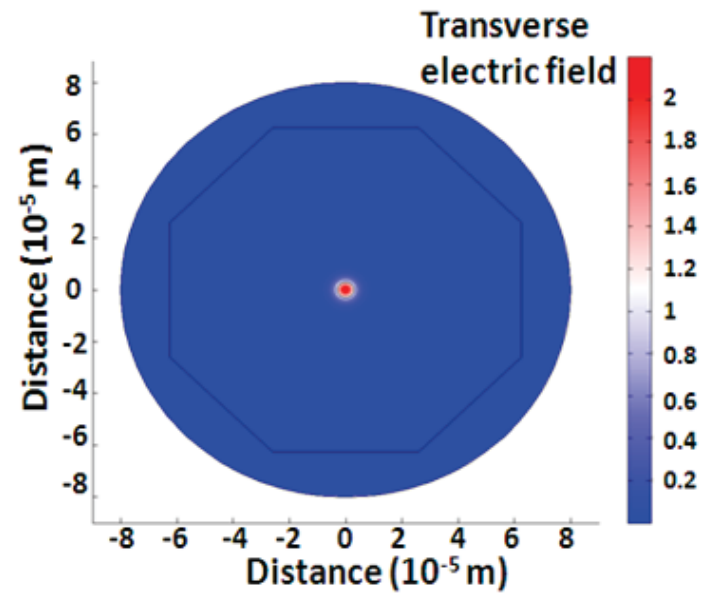

Fig. 2 Transverse electric field profiles of the normalized $\mathrm{HE}_{11}$ guided mode at the wavelength $\lambda_{\mathrm{s}}=1545 \mathrm{~nm}$ for the fiber $\mathrm{J}$.

These models are used to optimize the performance of these amplifiers in terms of gain and noise figure [17], [18]. Such optimization is based on the identification of the optimal amplifier design parameters (active fiber length, pumping scheme, RE concentration, refractive-index, geometrical parameters of the fiber transversal section) that maximize the amplification around $1550 \mathrm{~nm}$. However, as the characteristics of the fiber will change with radiations, it can be clearly seen that such calculation tools, including radiation effects, may also be used to enhance the amplifier performance not only before irradiation but also, for example, for a space mission by a more judicious choice of these "optimal" parameters. Furthermore, such an optimization of the amplifier can only be performed through simulations as it will require a too large number of samples and radiation experiments to identify the optimal system at reasonable costs.

A computer code that solves the general evolution equations describing the longitudinal propagation of pump, signal, forward and backward amplified spontaneous emission (ASE) in diversely doped RE optical fibers has been developed at Politecnico di Bari. Complete description of this simulation tool is out the scope of this paper but more details can be found in the following references [17] [19] [20]. Modeling the amplifier behavior involves different simulation steps. The first step determines, by using a full-vector finite element method (FEM) based-code, the spatial distribution of the fundamental guided mode corresponding to the $1545 \mathrm{~nm}$ transmitted signal to be amplified. This is achieved by considering the specific structure and composition features of both I and $\mathrm{J}$ fibers like their refractive index profiles, numerical apertures, core and inner cladding sizes. Figure 2 depicts the modulus of the normalized electric field $\mathrm{HE}_{11}$ for the fundamental mode at $\lambda_{s}=1545 \mathrm{~nm}$ evaluated by considering the fiber's J parameters.

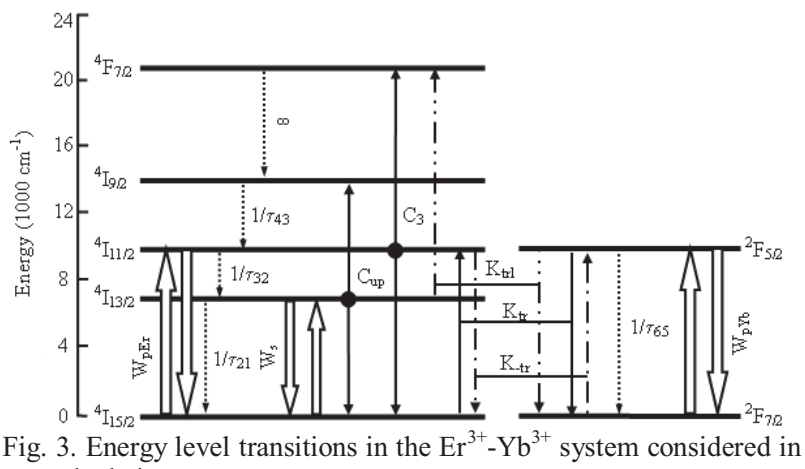

Fig. 3. Energy
our calculations

A particle swarm optimization approach has been used in Bari (see [21] for a description of this calculation method) to recover the main parameters of RE-doped amplifiers. The considered fibers are doped with different amounts of the $\mathrm{Er}^{3+}$ and $\mathrm{Yb}^{3+}$ active ions that permit the signal amplification. The modeling of the amplifier takes into account the dynamics of populations associated with their different energy levels under the influence of the injected pump and the signal to be amplified. Pump energy at the wavelength $\lambda_{\mathrm{p}}=915 \mathrm{~nm}$ is absorbed, in main part, by the $\mathrm{Yb}^{3+}$ ions which are in the ${ }^{2} \mathrm{~F}_{7 / 2}$ ground level and excited to the ${ }^{2} \mathrm{~F}_{5 / 2}$ level. Excited ytterbium ions transfer their energy to the nearby ground level $\mathrm{Er}^{3+}$ ions which are caused to transit to the ${ }^{4} \mathrm{I}_{11 / 2}$ level. The $\mathrm{Er}^{3+}$ ions in the ${ }^{4} \mathrm{I}_{11 / 2}$ level, by a non radiative decay, rapidly relax to the ${ }^{4} \mathrm{I}_{13 / 2}$ metastable level and this facilitates the population inversion phenomenon. The transition ${ }^{4} \mathrm{I}_{13 / 2} \rightarrow{ }^{4} \mathrm{I}_{15 / 2}$, close to the signal wavelength $\lambda_{\mathrm{s}}=1545 \mathrm{~nm}$ allows the amplifier gain via the stimulated emission. Unfortunately, two excited erbium ions on the ${ }^{4} \mathrm{I}_{13 / 2}$ level can exchange energy between them; one ion returning in the erbium ground state, by a non radiative transition, while the other raising to the ${ }^{4} \mathrm{I}_{9 / 2}$ excited level. A similar phenomenon occurs between two ions of the ${ }^{4} \mathrm{I}_{11 / 2}$ level, the former results in ${ }^{4} \mathrm{~F}_{7 / 2}$ level and the latter returns in the ground state. In addition, we consider also the secondary energy transfer from $\mathrm{Yb}^{3+}$ ions in the level ${ }^{2} \mathrm{~F}_{5 / 2}$ to the $\mathrm{Er}^{3+}$ ions in the ${ }^{4} \mathrm{I}_{13 / 2}$, and the $\mathrm{Er}^{3+}$ ions clustering effects.

\section{SIMULATION AND EXPERIMENTAL RESULTS ON FIBERS AND AMPLIFIERS}

\section{A. Validation of the Calculation Codes on Non-Irradiated Fibers and Amplifiers}

Some of the input parameters necessary for our calculations are extracted from measurements made on $\mathrm{I}$ and $\mathrm{J}$ fiber samples. Other ones are not yet directly measurable by our research group (eg. the lifetimes, energy transfer coefficients $\mathrm{K}_{\text {tr }}$ and $\mathrm{K}_{\text {trl }}$, upconversion coefficients $\mathrm{C}_{\text {up }}$ and $\mathrm{C}_{3}$ ) and have been recovered by means of the PSO approach. The erbium absorption cross sections have been evaluated directly from the spectral attenuation measurements performed on fiber I 
and $\mathrm{J}$ samples before irradiation, through excitation of the fiber core, according to the relation [18]:

$\sigma_{a}^{E r} \quad \lambda=\frac{a t t^{E r}(\lambda)}{10 \log e N_{E r} \Gamma_{s}}$

where att $\lambda$ is the erbium spectral attenuation expressed in $\mathrm{dB} / \mathrm{m}, N_{E r}$ is the erbium ion concentration in I (or $\mathrm{J}$ ) sample (ions $\left./ \mathrm{m}^{3}\right), \Gamma_{\mathrm{s}}$ is the overlap factor between the propagated mode and the erbium ion distribution defined as:

$\Gamma_{s}=\int_{0}^{2 \pi} \int_{0}^{D / 2}|E r, \phi|^{2} r d r d \phi$

where $\mathrm{D}$ is the diameter of the fiber core and $E(r, \phi)$ is the transverse electric field envelope normalized so that the surface integral of $|E|^{2}$ is equal to one.

The ytterbium absorption cross sections have been calculated by using the corresponding relation:

$\sigma_{a}^{Y b} \lambda=\frac{a t t^{Y b}(\lambda) S_{\text {clad }}}{10 \log e N_{Y b} S_{c o r e}}$

where $\mathrm{S}_{\text {core }}$ and $\mathrm{S}_{\text {clad }}$ are the core and cladding areas, respectively. The two different expressions calculating $\sigma_{a}^{E r}$

and $\sigma_{a}^{Y b}$ take into account the different pumping schemes core and DC pumping for $\mathrm{Er}^{3+}$ and $\mathrm{Yb}^{3+}$ absorption cross sections, respectively. Tested amplifiers based on I and $\mathrm{J}$ fibers have been designed in a backward pumping scheme [14]. The erbium emission cross sections have been calculated by using the McCumber theory [18]:

$\sigma_{e}^{E r} \lambda=\sigma_{a}^{E r} \lambda \exp \left[\frac{E_{Z L}-h \nu}{\kappa T}\right]$

where $E_{Z L}$ is the energy difference between the bottom of the ${ }^{4} \mathrm{I}_{13 / 2}$ manifold and the bottom of the ground state ${ }^{4} \mathrm{I}_{15 / 2}, T$ is the temperature and $\kappa$ is the Boltzmann's constant. Fig. 4 depicts the calculated erbium emission cross sections for both fibre I and fibre $\mathrm{J}$ evaluated by considering $E_{Z L}=0.81 \mathrm{eV}$ approximately the energy where the absorption peak occurs. This result has been comforted by experimental characterization of the two RE-doped fibers.

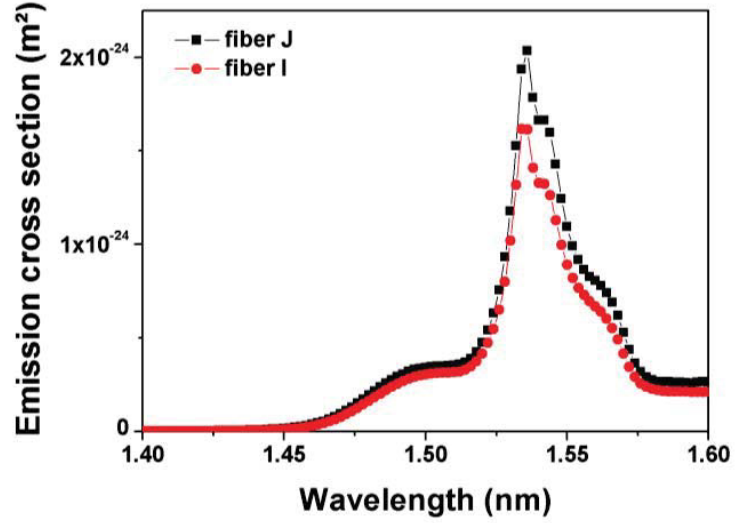

Fig. 4. Calculated erbium emission cross section for I and J fibers.

Fig. 5 compares the calculated output power at $1545 \mathrm{~nm}$ versus the input $915 \mathrm{~nm}$ pump current for amplifiers A\#1 and A\#2 with experimental results. These calculations have been done by considering the fiber parameters listed in Table I and those recovered by using the PSO approach listed in Table II. By considering that the transition rates due to cooperative upconversion, spontaneous emissions and first and secondary $\mathrm{Yb}^{3+}$ to $\mathrm{Er}^{3+}$ energy transfer depend on the input pump power, a suitable fitness function has been constructed and minimized by taking into account the experimentally measured signal output power values illustrated in Fig. 5.

TABLE I SPECTROSCOPIC PARAMETERS OF BOTH FIBRE I AND FIBRE $\mathbf{J}$

\begin{tabular}{c|c}
\hline PARAMETER & VALUE \\
\hline$\sigma_{\mathrm{aYb}} @ 915 \mathrm{~nm}$ fiber I & $0 \mathrm{~m}^{2}$ \\
$\sigma_{\mathrm{eYb}} @ 915 \mathrm{~nm}$ Fiber I & $7.3 \times 10^{-25} \mathrm{~m}^{2}$ \\
$\sigma_{\mathrm{aYb}} @ 915 \mathrm{~nm}$ Fiber J & $7.7 \times 10^{-25} \mathrm{~m}^{2}$ \\
$\sigma_{\mathrm{eYb}} @ 915 \mathrm{~nm}$ Fiber J & $0 \mathrm{~m}^{2}$ \\
$\tau_{32}\left({ }^{4} \mathrm{I}_{11 / 2} \rightarrow{ }^{4} \mathrm{I}_{13 / 2}\right)$ & $1 \mu \mathrm{s}$ \\
$\tau_{43}\left({ }^{4} \mathrm{I}_{9 / 2} \rightarrow{ }^{4} \mathrm{I}_{11 / 2}\right)$ & $0.1 \mu \mathrm{s}$ \\
$\mathrm{N}_{\mathrm{Er}}$ fibre I & $5.2 \times 10^{24}$ ions $/ \mathrm{m}^{3}$ \\
$\mathrm{~N}_{\mathrm{Er}}$ fibre J & $5.6 \times 10^{24}$ ions $/ \mathrm{m}^{3}$ \\
$\mathrm{~N}_{\mathrm{Yb}}$ fibre I & $1.1 \times 10^{26}$ ions $/ \mathrm{m}^{3}$ \\
$\mathrm{~N}_{\mathrm{Yb}}$ fibre J & $1.15 \times 10^{26}$ ions $/ \mathrm{m}^{3}$ \\
\hline
\end{tabular}

TABLE II. PSO RECOVERED PARAMETERS FOR BOTH FIBERS I AND J

\begin{tabular}{c|c|c}
\hline PARAMETER & FIBER I & FIBER J \\
\hline$\tau_{21}\left({ }^{4} \mathrm{I}_{13 / 2} \rightarrow{ }^{4} \mathrm{I}_{15 / 2}\right)$ & $7.2 \mathrm{~ms}$ & $8.0 \mathrm{~ms}$ \\
$\tau_{65}\left({ }^{2} \mathrm{~F}_{5 / 2} \rightarrow{ }^{2} \mathrm{~F}_{7 / 2}\right)$ & $1.0 \mathrm{~ms}$ & $0.8 \mathrm{~ms}$ \\
$\mathrm{Cup}$ & $4.3 \times 10^{-23} \mathrm{~m}^{3} \mathrm{~s}^{1}$ & $4.0 \times 10-^{-23} \mathrm{~m}^{3} \mathrm{~s}^{-1}$ \\
$\mathrm{C}_{3}$ & $5.6 \times 10^{-23} \mathrm{~m}^{3} \mathrm{~s}^{1}$ & $4.1 \times 10-^{23} \mathrm{~m}^{3} \mathrm{~s}^{-1}$ \\
$\mathrm{~K}_{\text {tr }}$ & $4.3 \times 10^{-22} \mathrm{~m}^{3} \mathrm{~s}^{1}$ & $4.0 \times 10-^{-22} \mathrm{~m}^{3} \mathrm{~s}^{-1}$ \\
$\mathrm{~K}_{\text {tr1 }}$ & $6.0 \times 10^{-23} \mathrm{~m}^{3} \mathrm{~s}^{1}$ & $7.0 \times 10-^{23} \mathrm{~m}^{3} \mathrm{~s}^{-1}$ \\
\hline
\end{tabular}



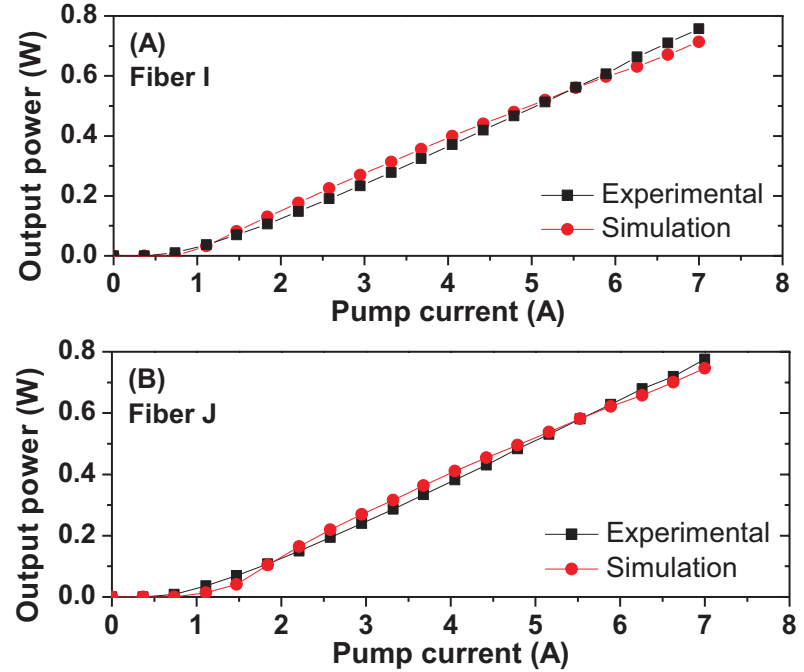

Fig.5. $1545 \mathrm{~nm}$ signal output power versus input pump current for (A) A\#1 and (B) A\#2 amplifiers based on non-irradiated fibers I and $\mathrm{J}$, respectively. Simulation results have been obtained based on Tables I and II input parameters and FEM results (see Fig.2).

As showed in Fig.5, our PSO optimization reproduces well the fiber amplifier performance before irradiation, confirming that the parameters list of Table I and Table II are correct.

\section{B. Radiation Response of the RE-doped Optical Fibers and Amplifiers}

In situ experiments [14] reveal a good behaviour of $\mathrm{A \# 1}$ amplifier based on fiber I compared to the responses of other amplifiers discussed in literature [9-12]. After a total dose of $\sim 40 \mathrm{krad}$, the output power of the amplifier signal at $1545 \mathrm{~nm}$ (with a 7A input pump current) decreases from $\sim 760 \mathrm{~mW}$ to $\sim 250 \mathrm{~mW}$ corresponding to a gain decrease from $\sim 19 \mathrm{~dB}$ to $\sim 13 \mathrm{~dB}$. The amplifier $\mathrm{A} \# 2$ based on fiber $\mathrm{J}$ presents an excellent radiation tolerance up to doses of $\sim 100 \mathrm{krad}$ with a gain decrease from $\sim 19 \mathrm{~dB}$ to $\sim 18 \mathrm{~dB} \quad(\sim 780 \mathrm{~mW}$ to $\sim 680 \mathrm{~mW}$ ) at the same pump power.

Post mortem experiments on samples of fibers I and $\mathrm{J}$ irradiated at different doses highlight the radiation-induced changes inside the two active fibers. As expected from amplifier tests, strong differences induced by the Ce-codoping are seen in their radiation sensitivities. Fig.6 and Fig.7 illustrate the evolution of the background attenuation with dose (see $\S$ II.A for description of the experimental setup) for the two fibers.

In Fig. 6, results provide evidence for a strong increase of the global attenuation (pre-irradiation attenuation plus radiationinduced attenuation (RIA)) in the whole studied range of wavelengths. This loss increasing is related to the generation of radiation-induced point defects in the glass matrix. From our previous work [8], we can assume that induced losses are mainly caused by phosphorus-related point defects. Some of the point defects are known [22], like Phosphorus-Oxygen Hole centers (POHC) with their absorption bands peaking at

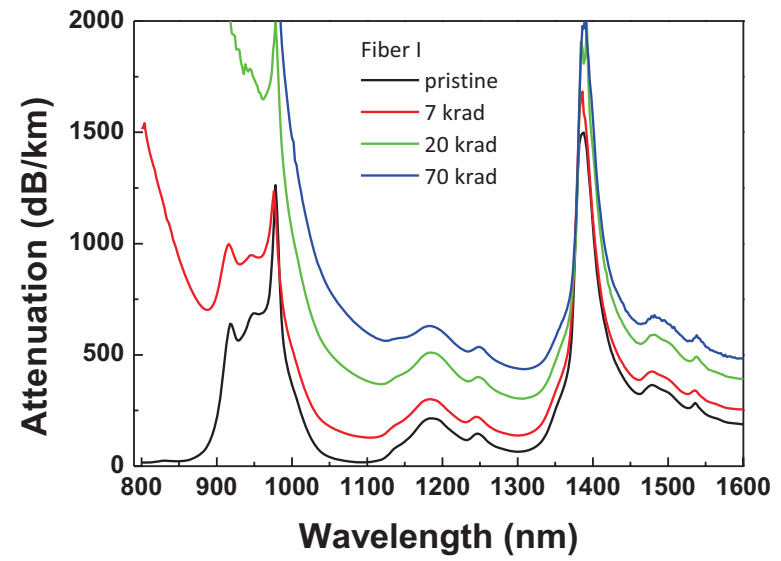

Fig. 6. Background attenuation measured in fiber I through excitation of the Double Clad (DC) of non-irradiated and irradiated samples at $7 \mathrm{krad}, 20 \mathrm{krad}$. and $70 \mathrm{krad}$.

around at $400 \mathrm{~nm}, 510 \mathrm{~nm}$ and $570 \mathrm{~nm}$. In the infrared domain, P1 defects are associated with an absorption band around $1.6 \mu \mathrm{m}$ that strongly impacts the $1545 \mathrm{~nm}$ signal [8]. Furthermore, it has also been shown on passive P-doped fibers that some of the P-related defects have still to be identified in this spectral range [23]. Comparing Fig.6 and Fig.7, it appears evident that the Ce-codoping of the glass matrix leads to the disappearance of the excess attenuation associated with Pdefects. Today, we assumed that this very positive influence is explained by the competition in the trapping of the charges released by irradiation in the matrix between $\mathrm{P}$ and Ce-related species. This will be more investigated in the future on the basis of spectroscopic measurements.

For the simulation part of our study, we mainly focused the validation of the implementation of radiation effects in our PSO calculations on the basis of the I fiber and A\#1 amplifier as the changes observed in $\mathrm{J}$ fiber and $\mathrm{A \# 2}$ are too limited to authorize precise characterization.

\section{Implementation of the Radiation Effects in the Calculation Procedure}

Changes in the attenuation values due to irradiation have been evaluated through different classes of post mortem measurements on samples of I and $\mathrm{J}$ fibers irradiated at three dose steps: $7 \mathrm{krad}$; 20 and $70 \mathrm{krad}$. Systematically, we have recorded the changes occurring at the pump wavelengths (around $915 \mathrm{~nm}$ ) and the emission wavelength (around $1545 \mathrm{~nm}$ ) in terms of attenuation.

Fig. 8 shows the measured changes in the spectral attenuation of fiber I around the pump wavelengths at the different doses with a multimode excitation. This excitation differs from the one used for Figs. 1, 6 and 7 in the sense that a shorter length of the active fiber was used for the measurements allowing to more precisely characterize the high absorption peaks related to rare earth ions at the detriment of background losses. 


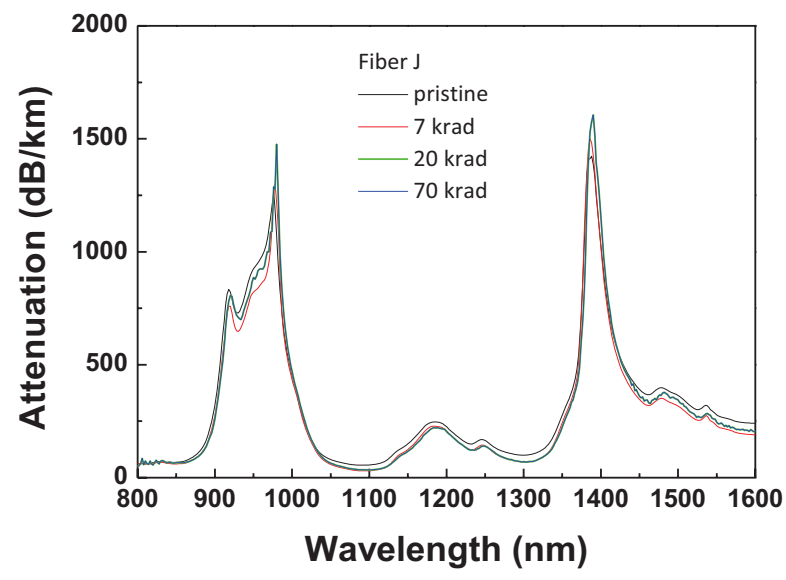

Fig. 7. Background attenuation measured in fiber J through excitation of the Double Clad (DC) of non-irradiated and irradiated samples at $7 \mathrm{krad}, 20 \mathrm{krad}$ and $70 \mathrm{krad}$.

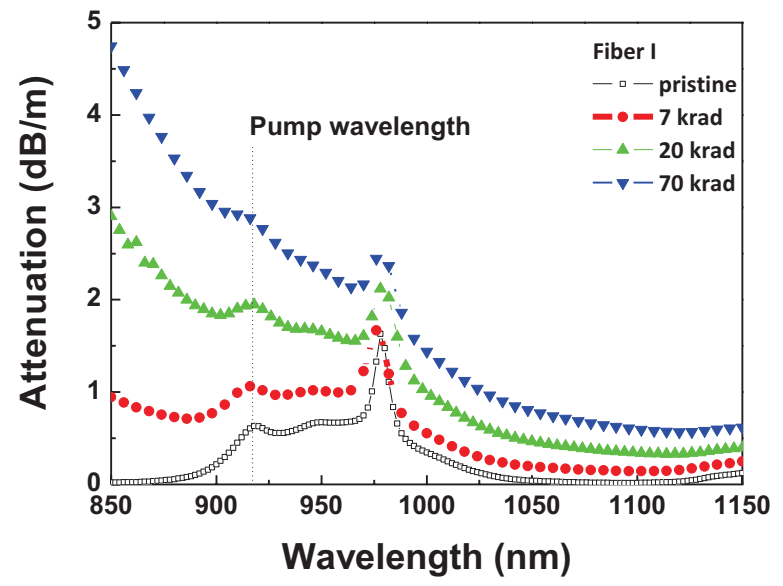

Fig.8. Evolution of the spectral attenuation measured around the pump wavelengths of $\mathrm{Yb}^{3+}$ ions in pristine sample and samples irradiated at 7, 20 and $70 \mathrm{krad}$ of the I fiber.

Then, the extracted values of attenuation are used to recalculate the amplifier behavior by considering new values of i) $\mathrm{Yb}^{3+}$ absorption cross sections at the pump wavelength, ii) $\mathrm{Er}^{3+}$ emission and absorption cross section curves, iii) background losses of the optical fiber, $\alpha$, at both pump and signal wavelengths, and by considering that the other spectroscopic properties of rare-earth ions will not be affected by irradiation. This hypothesis seems reasonable from our previous work $[1,8]$ but needs to be verified in the future through appropriate spectroscopic experiments on irradiated samples. As amplifier A\#1, based on fiber I, is more sensitive to irradiation, we will only present our results on this amplifier to highlight the potential of our simulation tool. Simulations of comparable quality have been done for the J-based amplifier. Table III reviews the differences between the input parameters before and at the different irradiation doses for the I and $\mathrm{J}$ fibers.

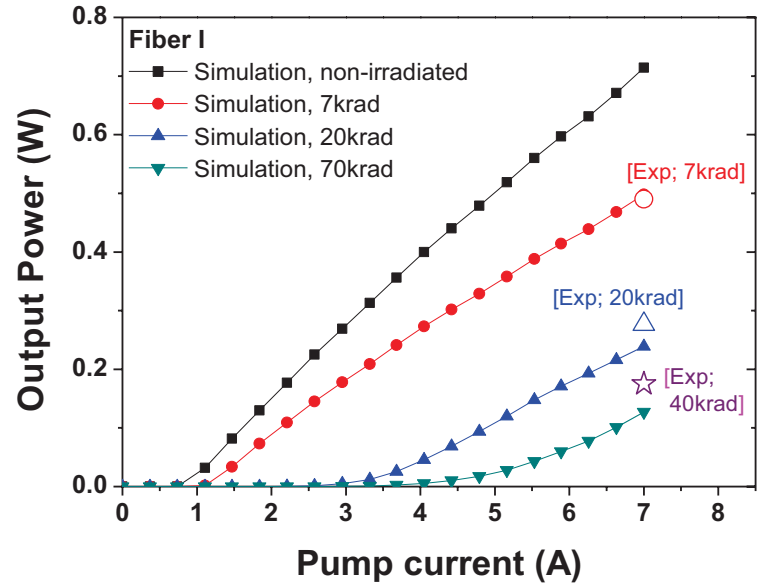

Fig.9. Comparison between the calculated $915 \mathrm{~nm}$ pump current dependence of the $1545 \mathrm{~nm}$ output power of amplifier A\#1 based on I fiber and the measured decrease of the output power measured in situ during irradiation of A\#1 operated at a pump current of 7A.

TABLE. III. NEW INPUT PARAMETERS EXTRACTED FROM THE IRRADIATED SAMPLES OF I AND J FIBERS AND USED FOR SIMULATION

\begin{tabular}{|c|c|c|c|c|}
\hline $\begin{array}{l}\text { PARAMETER } \\
\end{array}$ & PRISTINE & 7 KRAD & $20 \mathrm{KRAD}$ & $70 \mathrm{KRAD}$ \\
\hline $\mathrm{I}-\sigma a \mathrm{Yb}^{*}$ & $7.3 \times 10^{-25}$ & $5.7 \times 10^{-25}$ & $5.7 \times 10^{-25}$ & $5.3 \times 10^{-25}$ \\
\hline $\mathrm{J}-\sigma a Y b^{*}$ & $7.7 \times 10^{-25}$ & $6.8 \times 10^{-25}$ & $6.2 \times 10^{-25}$ & $6.2 \times 10^{-25}$ \\
\hline I- $\alpha-915^{* *}$ & 0.1 & 0.5 & 1.5 & 2.4 \\
\hline $\mathrm{J}-\alpha-915^{* *}$ & 0.1 & 0.11 & 0.1 & 0.12 \\
\hline $\mathrm{I}-\alpha-1545^{* * *}$ & 0.2 & 0.26 & 0.41 & 0.51 \\
\hline $\mathrm{J}-\alpha-1545^{* * *}$ & 0.23 & 0.24 & 0.25 & 0.26 \\
\hline
\end{tabular}

\begin{tabular}{l|cc}
$\mathrm{J}-\alpha-1545^{* * *}$ & 0.23 & 0.24 \\
\hline *Estimated at $915 \mathrm{~nm}\left(\right.$ expressed in $\left.\mathrm{m}^{2}\right)$
\end{tabular}

** Estimated at $915 \mathrm{~nm}$ (expressed in $\mathrm{dB} / \mathrm{m}$ )

*** Estimated at $1545 \mathrm{~nm}$ (expressed in $\mathrm{dB} / \mathrm{m})$

Fig.9. compares the simulation results taking into account the radiation-induced attenuation (RIA) changes in the fiber I on the A\#1 performance with measured results under irradiation of this amplifier [14]. An important remark is that in situ measurements of the amplifier response under irradiation can be compared with simulations based on values extracted on irradiated fibers several days after irradiation because it was checked that no or very limited bleaching of amplifier degradation exists after irradiation at room temperature.

This comparison shows that the calculations adequatly reproduce the general behavior of the amplifier (same kind of results for $\mathrm{A \# 1)}$ at $7 \mathrm{~A}$, validating the interest of such simulations for our study. This model, taking into account only the effects of radiation-induced attenuation adding to the pre-existing losses, appears sufficient to correctly reproduce the amplifier radiation response. However, it still need to be improved as the pump current dependence of the output power differs between simulations and experiments (see [14]). This will be achieved in the future to authorize more efficient simulations. 


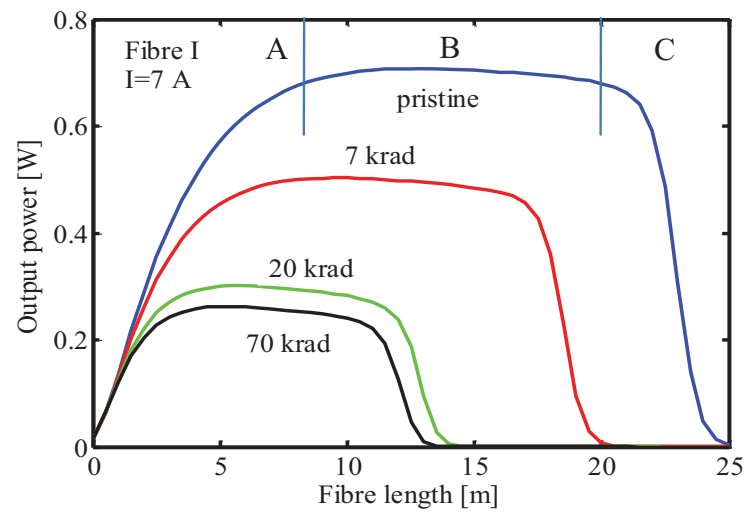

Fig.10. Comparison between the length dependence of the A\#1 $1545 \mathrm{~nm}$ output power calculated for the pristine amplifier and the ones made with fiber I irradiated at 7,20 and $70 \mathrm{krad}$. See text for the definition of the different zones.

\section{DISCUSSION}

The used code correctly reproduces the behavior of our amplifiers under irradiations. In this section, we discuss how such a simulation tool could help to develop more radiation tolerant amplifiers by adjusting their design. First, we discuss the influence of the choice of the RE-doped fiber length on the amplifier radiation sensitivity. Second, we compare for a same fiber, the relative expected degradation of amplifiers based on a forward pumping scheme instead of the tested backward pumping one. Finally, from a more fundamental point of view, we compare the relative influence of induced losses at pump and signal wavelengths. This will help in identifying the more influent point defects on the amplifier degradation and then give valuable indications for the future improvement of the fiber radiation tolerance.

\section{A. Influence of the RE-Doped Fiber Length}

The complex spectroscopic system, leading to the signal amplification by the pumping of the rare-earth ions, results from the competition between different emission and absorption mechanisms at different wavelengths. This explains that the amplifier output power at $1545 \mathrm{~nm}$ (or gain efficiency) will strongly depend on the length of the active fiber used for its design. This dependence is illustrated in Fig. 10 for nonirradiated fiber I and for this fiber irradiated at $7 \mathrm{krad}, 20 \mathrm{krad}$ and $70 \mathrm{krad}$.

Before irradiation, the output power of an amplifier based on this fiber will first increase with the number of active ions (then the RE fiber length) as the contribution of these centers to the signal amplification dominates their induced absorption at the signal wavelength (Zone A in Fig.8). For a range of fiber lengths, these mechanisms compensate themselves, resulting in a stable output power with length (Zone B). For longer fiber lengths, the signal absorption due to the whole fiber length dominates the RE emission and the output power will then abruptly decrease with the fiber length (Zone C).

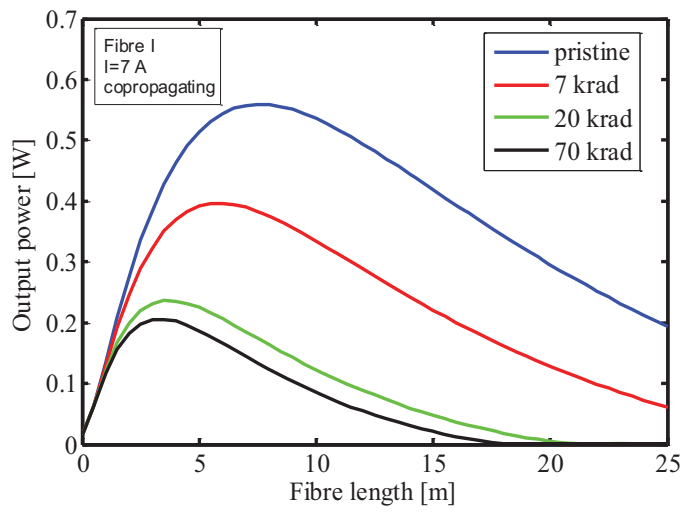

Fig.11. Comparison between the length dependence of the A\#1 $1545 \mathrm{~nm}$ output power calculated for the pristine amplifier designed with a forward pumping scheme and for the ones made with fiber I irradiated at 7, 20 and $70 \mathrm{krad}$.

Simulations also reveal that radiations impact the amplifier efficiency by two possible ways. At low doses, radiations lead to induced attenuation that, for an optimized length before irradiation, decrease the $1545 \mathrm{~nm}$ output power, without changing the fact that the used fiber length remains optimal under these new conditions. At higher doses, these excess losses can lead to the absorption of the whole pump at $915 \mathrm{~nm}$ before the end of the active fiber. Inside the remaining part of the active fiber that is not pumped, the $1545 \mathrm{~nm}$ will no more by amplified but will still be absorbed by the $\mathrm{Er}^{3+}$ ions, resulting in a very quick decrease of the fiber amplifier efficiency in this configuration. This additional way of degradation may explain the complex degradation observed in radiation-hardened amplifiers at high doses, with a sudden increase of the degradation at a certain dose level.

\section{B. Influence of the Amplifier Scheme}

PSO simulations can also be a very efficient way to evaluate the potential of different amplifiers designs for use in space. We can distinguish between two classes of amplifiers. In backward scheme, the $1545 \mathrm{~nm}$ signal is propagating in the inverse direction of the pump laser at $915 \mathrm{~nm}$. Such a scheme was used for the amplifiers tested under radiations as it allows to obtain higher $1545 \mathrm{~nm}$ gain for a given pump power at $915 \mathrm{~nm}$. Amplifiers can also be designed in a way where pump and signal propagate along the same direction, called forward pumping scheme. Fig. 11 illustrates the length dependence of the $1545 \mathrm{~nm}$ for this amplifier design in the case of pristine or irradiated I fiber.

The length dependence of the A\#1 output power differs from the one associated with the backward pumping scheme. In the backward pumping the amplified signal efficiently extracts power from the pump because the ${ }^{4} \mathrm{I}_{13 / 2}$ ion population is more inverted at the fiber end. As result, this pumping scheme is more efficient than the forward one. On the contrary, longer optimal lengths are needed for backward pumping. 


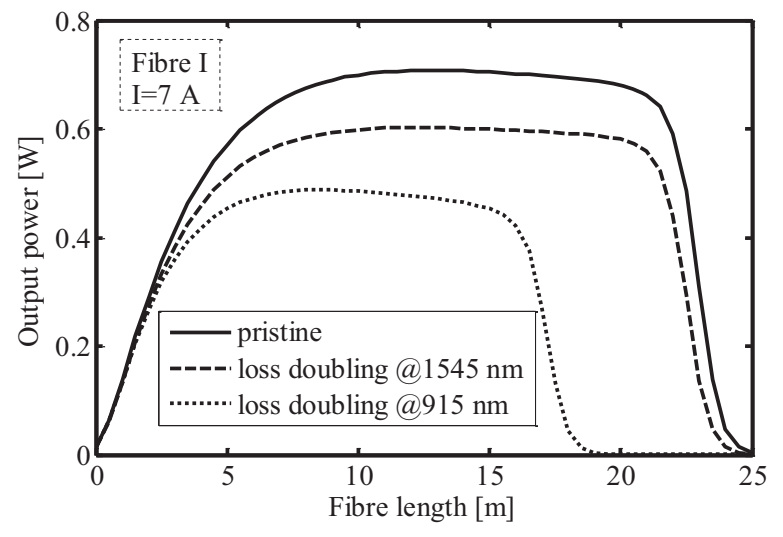

Fig.12. Comparison between the length dependence of the A\#1 $1545 \mathrm{~nm}$ output power calculated for the pristine amplifier in a backward pumping scheme based on fiber I and by considering loss doubling at $915 \mathrm{~nm}$ and $1545 \mathrm{~nm}$ respectively.

However, for this case, changing the pumping scheme will not authorize to improve the radiation hardness of the amplifier based on fiber J.

\section{Relative Influence of Radiation-Induced Losses at the Pump and Emission Wavelengths}

From our study, it appears that optimizing the composition of the fiber core and the fiber length are two efficient ways to improve the radiation tolerance of fiber-based amplifiers. Simulation tools can also be used to identify the most influent point defects that lead to the observed A\#1 degradation under radiations. In Fig. 12, we compare the effects of doubling the pump losses at $915 \mathrm{~nm}$ without changing the $1545 \mathrm{~nm}$ and doubling the IR losses without affecting the pump propagation.

From this figure, it clearly appears that the losses at $915 \mathrm{~nm}$ have a stronger impact on the amplifier behavior. Losses around the emission wavelength decrease the global efficiency of the amplifier without changing the optimization choices made before irradiation. At the opposite, induced losses around the pump wavelengths affect strongly the designer choice for the amplifier. As a consequence, future studies must be focused to reduce the RIA around the pump wavelength of amplifiers.

\section{CONCLUSION}

We present, in this paper, a coupled approach based on experiments and simulations that can be used to improve the radiation tolerance of active fibers and fiber-based amplifiers. We validate this approach by studying Er/Yb-doped fibers that were previously identified as the most promising candidates for operation in harsh environments. An excellent improvement of the radiation tolerance of the $\mathrm{Er} / \mathrm{Yb}$ amplifiers has been achieved by working on the design of the active fiber. The use of a radiation-hardened optical fiber, based on an active fiber with core codoped with Phosphorus, Cerium, Ytterbium and Erbium, allows a strong decrease of the radiation effects on the fiber-based amplifiers. Simulation tools including Particle Swarm Optimization (PSO) procedure permit to reproduce the amplifier behavior before and after irradiation. A this time, only radiation effects on the attenuation properties of the active fibers are implemented in the code but further experiments/simulations will be done to also model the radiation induced changes in the spectroscopic properties of the rare earth ions. By playing with the code, we evaluated the impact of the fiber design on its radiation response. Clearly, our analysis showed that the fiber length of the amplifier should not be optimized before irradiation, as usually done, but this fiber length must be chosen to reduce the impact of the radiation-induced losses around the pump wavelengths that strongly impact the fiber response in both backward and forward pumping scheme.

\section{REFERENCES}

[1] S. Girard, B. Tortech, E. Regnier, M. Van Uffelen; A. Gusarov, Y Ouerdane, J. Baggio, P. Paillet, V. Ferlet-Cavrois, A. Boukenter, J-P. Meunier, F. Berghmans, J.R. Schwank, M.R. Shaneyfelt, J.A. Felix, E.W. Blackmore, H. Thienpont « Proton- and gamma-induced effects on erbium- doped optical fibers", IEEE Transactions on Nuclear Sciences, vol. 54, n6, pp. 2426-2434, 2007.

[2] G.M. Williams, M.A. Putnam, C.G. Askins, M.E. Gingerich, E.J Friebele, "Radiation effects in erbium-doped optical fibres", IEE Electronic Letters 28, pp. 1816-1818, 1992.

[3] H. Henschel, O. Kohn, H.U. Schmidt, J. Kirchof, S. Unger, "Radiationinduced loss of rare earth doped silica fibres", IEEE Transactions on Nuclear Sciences, vol. 45, n³, pp. $1552-1557,1998$.

[4] M. Ott, "Radiation Effects expected for Fiber Laser/Amplifier and Rare-Earth doped Optical Fibers", NASA GSFC, Parts, Packaging and Assembly Technologies Office Survey Report, 2004.

[5] G.M. Williams, E.J. Friebele, "Space radiation effects on erbium-doped fiber devices: sources, amplifiers, and passive measurements", IEEE Transactions on Nuclear Sciences, vol. 45, n³, pp. 1531 - 1536, 1998.

[6] B.P. Fox, K. Simmons-Potter, W.J. Thomes, D.A.V. Kliner, "GammaRadiation-Induced Photodarkening in Unpumped Optical Fibers Doped With Rare-Earth Constituents", IEEE Transactions on Nuclear Sciences, vol. 57, n³, pp. 1618 - 1625, 2010.

[7] B.P. Fox, K. Simmons-Potter; W. J. Thomes, Jr.; D. C. Meister; R. P. Bambha; and D.A.V. Kliner, "Temperature and dose-rate effects in gamma irradiated rare-earth doped fibers" SPIE 7095, 70950B, 2008.

[8] S. Girard, Y. Ouerdane, B. Tortech, C. Marcandella, T. Robin, B. Cadier, J. Baggio, P. Paillet, V. Ferlet-Cavrois, A. Boukenter, J-P. Meunier, J. R. Schwank, M.R. Shaneyfelt, P.E. Dodd, E.W. Blackmore "Radiation Effects on Ytterbium- and Ytterbium/Erbium-doped Double-Clad Optical Fibers" IEEE Transactions on Nuclear Sciences, vol. $56, \mathrm{n}^{\circ} 6$, pp. $3293-3299,2009$.

[9] T.S. Rose, D. Gunn, G.C. Valley, "Gamma and proton radiation effect in erbium-doped amplifiers: active and passive measurements", J. Light. Technol., vol. 19, pp. 1918-1923, 2001.

[10] M. Li, J. Ma, L. Y. Tan, Y.P. Zhou, S. Y. Yu, J.J. Yu, and C. Che, "Investigation of the irradiation effect on Erbium-doped fiber amplifiers composed by different density erbium-doped fibers », Laser Physics, 19(1), pp.138-142, 2009

[11] A. Gusarov, M. Van Uffelen, M. Hotoleanu, H. Thienpont, and F. Berghmans, "Radiation Sensitivity of EDFAs Based on Highly ErDoped Fibers", J. Light. Tech. 27(11), 1540-1545, 2009.

[12] M. Alam, J. Abramczyk, P. Madasamy, W. Torruellas, and A. Sanchez, "Fiber amplifier Performance in $\gamma$-radiation environment", OSA/Optical Fiber Conference 2007, paper OMF4.

[13] J. Ma, M. Li, L. Tan, Y. Zhou, S. Yu,Q. Ran, "Experimental investigation of radiation effect on erbium-ytterbium co-doped fiber 
amplifier for space optical communication in low-dose radiation environment", Optic Express, vol.17, n¹8, pp. 15571-15579, 2009.

[14] S. Girard, M. Vivona, A. Laurent, Y. Ouerdane, C.Marcandella, T

Robin, A. Boukenter, B. Cadier, " Radiation hardening techniques for $\mathrm{Er} / \mathrm{Yb}$ doped optical fibers and amplifiers for space applications", submitted for publication.

[15] M. Vivona, S. Girard, C. Marcandella, T. Robin, B. Cadier, M. Cannas, A. Boukenter and Y. Ouerdane, "Influence of Ce codoping and $\mathrm{H}_{2}$ preloading on $\mathrm{Er} / \mathrm{Yb}$-doped fiber: Radiation response characterized by Confocal Micro-Luminescence", Journal of non-crystalline solids, vol.357, pp.1963-1965, 2011.

[16] E. Yahel, A. Hardy, "Efficiency optimization of high-power, $\mathrm{Er}^{3+}$ $\mathrm{Yb}^{3+}$-codoped fiber amplifiers for wavelength-division-multiplexing applications", J. Opt. Soc. Am. B, vol. 20, pp. 1189-1197, 2003.

[17] M. De Sario, L. Mescia, F. Prudenzano, F. Smektala, F. Deseveday, V. Nazabal,J. Troles, L. Brilland, "Feasibility of $\mathrm{Er}^{3+}$-doped, $\mathrm{Ga}_{5}$ $\mathrm{Ge}_{20} \mathrm{Sb}_{10} \mathrm{~S}_{65}$ chalcogenide microstructured optical fiber amplifiers," Opt. \& Las. Technol., vol. 41,pp. 99-106, 2009.

[18] A. Bjarlev, "Optical fiber amplifiers: design and system application," Artech House, 1993.

[19] F. Prudenzano, L. Mescia, A. D’Orazio, M. De Sario, V. Petruzelli, A. Chiasera, and M. Ferrari, "Optimization and characterization of RareEarth doped Photonic-Crystal-Fiber Amplifier Using Genetic Algorithm”, J. Lightwave Technol., vol. 25 (8), pp. 2135-2141,2007.

[20] G. Fornarelli, L. Mescia, F. Prudenzano, M. De Sario, F. Vacca, "A neural network model of erbium-doped photonic crystal fibre amplifier", Optics and Laser Technology, vol. 41, pp. 580-585, 2009.

[21] A. Giaquinto, L. Mescia, G. Fornarelli, F. Prudenzano: "Particle swarm optimization-based approach for accurate evaluation of upconversion parameters in $\mathrm{Er}^{3+}$-doped fibers," Opt. Lett. vol. 36, pp. 142-144, 2011.

[22] D.L. Griscom, E.J. Friebele, K.J. Long, and J.W. Fleming, "Fundamental defect centers in glass: Electron spin resonance and optical absorption studies of irradiated phosphorus-doped silica glass and optical fibers," Journal of Applied Physics, vol. 54 (7), pp. 3743 $3762,1983$.

[23] E. Régnier, I. Flammer, S. Girard, F. Gooijer, F. Achten, and G. Kuyt. "Radiation-induced attenuation at IR wavelength in silica based optical fibers", IEEE Trans. Nucl. Sci. 54 1115-1119 (2007). 